\title{
Some Clinical Advances on Brugada Syndrome
}

\author{
Hu Kangxin ${ }^{1}$, Pan Jie ${ }^{2, ~ *, ~ Y a o ~ W e n l i a n g ~}{ }^{1}$ \\ ${ }^{1}$ Clinical Department, Nanchang City Institute of Medical Science, Nanchang, China \\ ${ }^{2}$ Literature Department, Nanchang City Institute of Medical Science, Nanchang, China \\ Email address: \\ hupei7758521@163.com (Hu Kangxin),panjie29@163.com (Pan Jie), 475644902@qq.com (Yao Wenliang)
}

\section{To cite this article:}

Hu Kangxin, Pan Jie, Yao Wenliang. Some Clinical Advances on Brugada Syndrome. Clinical Medicine Research. Vol. 4, No. 6, 2015, pp. 168-171. doi: 10.11648/j.cmr.20150406.11

\begin{abstract}
Brugada syndrome is an inherited arrhythmogenic disorder that exhibits ECG ST-segment elevation with a negetive T-wave in the right precordial leads $\left(\mathrm{V}_{1}-\mathrm{V}_{2}\right)$, with normal heart structure, predisposing to VF and SCD. Its symptoms include syncope, nocturnal agonal respiration and cardiac arrest. Recently, reserches on Brugada syndrome had archived some advances, for examples, regarding to exposuring concealed Brugada 1 type ECG, besides pharmacological provocation, there are two orter methods: putting right precordial lead on the second intercostal space or Holter monitoring; early repolarization(J-wave) has important value in prognostic judgment of Brugada syndrome; radiofrequency catheter ablation was generally applied only in epicardium of RVOT in the past, while it has been applied in endocardium of RVOT today. Therefore, the endocardium of RVOT can be considered as an alternative site in the treatment of recurrent VF in Brugada syndrome.
\end{abstract}

Keywords: Brugada Syndrome, Early Repolarization, Drug Provocation Test, Radiofrequency Catheter Ablation

\section{Introduction}

Brugada syndrome $(\mathrm{BrS})$ is an inherited arrhythmogenic disorder that exhibits ECG ST-segment elevation and a negetive T-wave in the right precordial leads (V1-V2), with normal heart structure, predisposing to VF and SCD. It is autosomal dominant inheritance with incomplete penetrance, whose onset time often around the age of $40 \mathrm{BrS}$ is more common in men than in women, probably due to the influence of hormone; while is rare in infants and children, probably due to their low testosterone levels [1]. The global prevalence rate is $5 \sim 20 / 10000$, and in Southeast Asia it is the local epidemic [1]. Its symptoms include syncope, nocturnal agonal respiration and SCD [caused by polymorphic ventricular tachycardia (PVT)/VF], which may occur after overeating, at rest or during sleep, probably due to vagotonia. But most patients are still asymptomatic [1].

\section{Some Diagnosing Problems}

\subsection{Brugada ECG Patterns [1]}

There are 3 types of ECG patterns: (1)Type 1: Electrocardiogram of right ventricular lead (V1-V2) shows coved ST-segment elevation $2 \mathrm{~mm}$, with the T-wave inversion, V1-V3 no equipotential separation; (2)Type 2: ST segment elevation, gradually becoming inferior oblique shape, followed by positive or bi-directional T-wave, saddle shape, J-wave amplitude higher than $2 \mathrm{~mm}$; (3)Type 3 : right ventricular lead ST segment elevation $\leq 1 \mathrm{~mm}$, coved shape or saddle, or both. By now, only type 1 ECG pattern has diagnostic value to $\mathrm{BrS}$, type 2 or 3 is not considered to be of any significance to the diagnosis. Even type 1 ECG pattern, due to the incomplete penetrance and the dynamic character of ECG, sometimes can be concealed. ECG diagnosis of BrS may face difficulties. For this there are 3 ways to overcome them.

\subsection{Methods to Reveal Concealed Type 1 ECG Pattern}

The following 3 methods are available for clinical use:

(1) Place ECG right ventricular lead high in the chest (until 2nd intercostal space) Antzelevitch and Nof [2] placed the right lead at high position(up to 2nd intercostal space), regardless of whether there is a drug challenge, the sensitivity of ECG determinating BrS phenotype increased. Because only in high intercostal space position, ECG recording is easy and noninvasive. So this method is recommended as the alternative method for sodium channel blockers provocation test revealing type 1 ECG pattern. Only when this method failed, drug provocation test is considered. But the generalization of type $1 \mathrm{ECG}$ diagnosing $\mathrm{BrS}$ caused by this method should also be taken into account. 
(2) 12-lead 24-hour Holter monitoring (12L-Holter) Cerrato, et al [3] found the diurnal fluctuation of BrS ECG, and type 1 ECG was common at 12 o'clock noon until $6 \mathrm{pm}$. They think type 1 ECG pattern is measured more often by 12L-Holter than by regular ECG habitual trace. This means 12L-Holter may cancel $20 \%$ patients with non-risk-free sodium channel blocker drug provocation test. Therefore, 12L-Holter should be considered as the first-line screening test.

(3) Drug provocation test According to the opinion of Antzelevitch and Brugada [4], the drugs and their dosage used in provocation test are as follows: Ajmaline: $1 \mathrm{mg} / \mathrm{kg} / 5 \mathrm{~min}$, intravenous; Flecainide: $2 \mathrm{mg} / \mathrm{kg} / 10 \mathrm{~min}$; Procainamide: $10 \mathrm{mg} / \mathrm{kg} / 10 \mathrm{~min}$; Pilacani: $1 \mathrm{mg} / \mathrm{kg} / 10 \mathrm{~min}$, intravenous. The commonly used drugs are Ajmaline and Flecainide in foreign countries and Propafenone in the domestic [5]. Now brief introductions are as follows: (A)Ajmaline: Considering the research results so far, Uzieblo-Zyczkowsk, et al [6] applied Ajmaline because of its highest sensitivity and specificity as well as the drastical reduce of complications in the same time test. Another reason to choose Ajmaline was its short half-life (near 10min), which was convenient for a short time post-test observation on patients in hospital not more than 1 day. Conte, et al [7] think that $1.8 \%$ of patients with this drug test can produce sustained supraventricular arrhythmias (sVAs), but none of them occurrs sudden cardiac death or further fatal ventricular arrhythmias later. (B) Flecainide: Compared with Ajmaline, its effect is slightly inferior. Because Ito is the basic mechanisms of pathogenesis of $\mathrm{BrS}$ [2], it is believed that the inhibition of Ito by Flecainide becomes the basis of this difference. Drubner, et al [8] used a single oral dose of $400 \mathrm{mg}$ Flecainide and suggested that BrS typical coved ECG pattern (type 1) do not occur atrioventricular block or atrial tachyarrhythmia. Calvo, et al [9] (2015) suggested that ECG changes have time dependence on the reaction of Flecainide. Recording for a long time (10 90min), the rate of type 1 ECG pattern has a progressive increases. However, there are some inadequacies in drug provocation test using class 1 antiarrhythmic specific drugs [6], for example, they can cause fatal arrhythmias induced by their arrhythmogenic effects, meanwhile, we should obtain the consent of patients of concealed BrS before the test. The indications about ending drug test [4] are: (1)the appearance type 1 Brugada ECG; (2) ST segment increase $\geq 2 \mathrm{~mm}$ in type 2 ECG pattern; (3) the occurrence of ventricular premature beats or other arrhythmia; (4)QRS widening $\geq 130 \%$ of the baseline. In the presence of atrial or ventricular conduction disease (suspected Lev-Lenegre disease or QRS widening, P-wave increase or PR interval prolonging), the application of sodium channel blockers should be particularly careful [4]. In individual cases, if there is a mechanical electrical separation, the use of isoprenaline and sodium lactate is effective.

\subsection{Brugada Phenocopy (BrP)}

In the diagnosis of $\mathrm{BrS}$, we should pay attention to the identification of $\mathrm{BrP}$. BrP is a clinical disease different from congenital $\mathrm{BrS}$ in the etiology [10]. BrP is characterized by right heart leading V1-V3 type 1 or type 2 Brugada ECG pattern. BrP showing type 2 ECG pattern is easy to identify, problems are in the distinguish of those showing type 1 ECG. For $\mathrm{BrP}$ is caused by various clinical situations, including acute myocardial infarction, variant angina [2, 4], right bundle branch block, early repolarization, acute myocardial ischemia, pericarditis, hypercalcemia, hyperkalemia, hypothermia, and primary right ventricular disease, especially arrhythmogenic right ventricular hypoplasia/cardiomyopathy(ARVD/C) [10], as well as pulmonary embolism [11] and dissecting aortic aneurysm [4]. Due to space limitations, the differential diagnosis of these $\mathrm{BrP}$ and $\mathrm{BrS}$ cannot be enumerated. Only the differential diagnosis with ARVD/C is described briefly as following table, and the original table has been minorly added and deleted.

Table 1. The differential diagnosis of $A R V D / C$ and $B r S$.

\begin{tabular}{lll}
\hline Clinical conditions & ARVD/C & BrS \\
\hline Age(Years) & $25 \sim 35$ & $35 \sim 40$ \\
Distribution & mainly in Italy & Worldwide, local endemic in South-East Asia \\
Genetics & $\mathrm{AD}(\mathrm{AR})$ & $\mathrm{AD}$ \\
Chromosome & $1,2,3,10,14$ & 3 \\
Gene & hRYR2, Plakobin, desmoplakin & SCN5A \\
Symptoms & Palpitation, syncope, cardiac arrest & syncope, nocturnal agonal respiration, cardiac arrest \\
Pathology & Full expansion of right ventricle, bulging or aneurysm & Without organic heart disease \\
ECG & Monomorphic ventricular tachycardia, often induced & Polymorphic ventricular tachycardia, often \\
& by Catecholamines or movement & occurring at rest or during sleep \\
\hline
\end{tabular}

\section{Relaitonship Among Brugada Syndrome, J-wave Syndrome and Early Repolarization}

The ECG change characteristics of BrS has been described above, and will not be repeated here. Early repolarization(ER) is characterized by J-point elevation, QRS terminal fuzzy, with or without ST-segment elevation, J-point and/or ST segment elevation $0.1 \mathrm{mV}$ from at least two adjacent lead baseline $[12,13]$. J-wave syndrome is an immediate deflection after the QRS wave on surface electrocardiogram. When $\mathrm{R}$ is covered partly, it seems to be J-wave elevation or ST-segment elevation [14]. Because of the significant features of J-wave forming $\mathrm{BrS}$ and early repolarization syndrome (ERS), both of BrS and ERS have the common Ito-mediated J-waves electrocardiographic findings, clinical consequences, 
risk factors and the same arrhythmia platform. Thus, Antzelevitch [12, 15] advocated that these syndromes should be classified under the heading of J-wave syndrome, although this 3 types are different in the wave amplitude and the lead placement sites of abnormal J-wave. It should be noted that J-wave $>0.1 \mathrm{mV}$ accompanied by decline or horizontal ST segment is a malignant ER mode [16], which can be differentiated from BrS in the ECG.

It should be emphasized that ER ( $\mathrm{J}$-wave) is of great value in the prognosis judgement of BrS. Kawata, et al [17] think that ER (permanent or intermittent) occurring in lower (II, III, aVF) and lateral (I, avL, V4-V6) lead is the independent predictor factor of fatal arrhythmia events for BrS. Talkaqi, et al [18] evaluated the value of J-wave and J-wave morphology in the prognosis of BrS. They found that patients with the lower or lateral lead J-wave or with horizontal ST segment elevation had higher rate of cardiac events than those without J-wave. Multivariate analysis showed that symptoms, the V2 lead QRS $>90 \mathrm{~ms}$ and the lower, lateral lead J-wave and/or horizontal ST segment morphology after J-wave are important to predict the heart accident of BrS.

Although the first expert consensus, BrS ECG diagnosis has been confirmed, because of the dynamic and concealed characteristics of ST segment, and the appearance of BrP, so the $\mathrm{BrS}$ diagnosis cannot solely rely on ECG. Confirmed diagnosis must be combined with one of the following 4 conditions [1, 2]: (1) family history, and family members younger than 45 have had sudden cardiac death or the family has type 1 ECG pattern; (2) arrhythmia related symptoms, such as syncope, and epileptic seizures and nocturnal agonal respiration; (3) ventricular arrhythmia as polymorphic ventricular tachycardia(PVT) or VF; (4)programmed electric stimulus can induce VT/VF.

\section{Some Progress in Treatment of BrS}

Until 2015, the unanimous view $[1,2,19,20]$ is: the countermeasures against $\mathrm{BrS}$ sudden cardiac death is the implantable cardioverter defibrillator (ICD). But ICD therapy is accompanied by inappropriate electrical shocks caused by ventricular bradycardia attack and instrument complications [20]. Because ICD has such shortcomings, it has been reported in the literature $[19,21]$ that radiofrequency catheter ablation method is used to treat inappropriate shock or ICD-rejecting patients of BrS with VF. Nedemanee, et al [22] noted that amplitude of abnormal ECG was higher in epicardial than that in endocardium of right ventricular outflow tract, so that radiofrequency catheter ablation was applied in epicardial location. In $89 \%$ of patients with BrS, ECG pattern had been mormalized. Sunsaneewitayakul, et al [23] noticed when using equipotential mapping, late activation zone(LAZ) as electrical activity was recorded from $\mathrm{J}$ point to RVOT endocardial surface ECG lead V1 and V2 $+60 \mathrm{~ms}$ interphase. Radiofrequency catheter ablation of endocardium LAZ was used to modify Brugada ECG pattern of VF storm patients. VF did not recur during the follow-up period. It seems to be considered that RVOT endocardial is an alternative location for radiofrequency catheter ablation treating recurrence of VF in patients with $\mathrm{BrS}$.

When it is not easy to get ICD areas or radiofrequency catheter ablation cannot be carried out, drug treatments are available: 1) Ito blockers: (1) Quinidine: The existence of significant Ito is the basic mechanisms of $\mathrm{BrS}$, so using quinidine is a wise "transitional treatment"("Bridge therapy"). The disadvantages of oral quinidine include gastrointestinal adverse reactions, delayed rectifier current (Ikr and Iks) quickly and slowly activated by quinidine block, easily occurring acquired long QT syndrome. Another point to mention is the efficacy of quinidine decreases if its dosage is too large. Because in high plasma concentrations, quinidine also blocks inward sodium current (INa), INa may fight the increases of repolarization spatial diffusion and the blocking effects of $\mathrm{Ikr}$ and Iks that trigger torsades de points(TdP). (2)Tedisamil: Without significant inward current block effect like quinidine, it may be more effective than quinidine. 2) Isoproterenol: This drug can reduce vagus nervous tension, increase heart rate, and thus help reduce Ito current. It is effective in acute and chronic treatment of patients with VF storm. 3) Cilostazol: a kind of phosphodiesterase III inhibitors. It has the potential to enhance calcium current (ICa), decrease the Ito secondary to the increase of heart rate, and can make the ST segment of BrS normalize and prevent VF. 4) Dimettyl lithospermate B (dmLSB) [21, 24]: It is extracted from salvia miltiorrhiza for traditional Chinese medicine herbal remedy. Fish, et al [24] reported that dmLSB slows INa inactivation, leading to the increase of early action potential inward currents, and has antiarrhythmic effects on BrS. It is worthy of further study and application of medical workers in China.

\section{References}

[1] Brugada R, Campuzano O, Serguella-Brugada G, et al. Brugada syndrome [J]. Methodist Debakeg Cardiovasc, 2014, 10(1):25-28 PMID 24932359.

[2] Antzelevitch C, Nof E. Burgada syndrome: Recent advance and controversies [J]. Curr Cardiol Rep, 2008, 10(5):376-383 PMC 2614235.

[3] Cerrato N, Givstetto C, Gribaude E, et al. Prevalence of type 1 Brugada electrocardiographic pattern evaluated by twelve lead twenty-four-hour Holter monitoring[J].Am J Cardiol, 2015,115(1):52-56 PMID 25446872.

[4] Antzelevitch C, Brugada P, Brugada J, et al. Brugada syndrome: From cell to bedside [J]. Curr Probl Cardiol, 2005, 30(1):9-54 PMC 1975801.

[5] Li CL, Hu DY. Cardiac channellopathy induced syncope.(in Chinese)][J]. Advances in Cardiovascular Diseases, 2006, 27(4):407-412.

[6] Uzieblo-Zyczkowsk B, Gielerak G, Michalkiewicz D. Usefullness of patients history and noninvasive electrocardiographic patterns in prediction of ajmaline test results in patients with suspected Brugada syndrome [J]. Arch Med Sci, 2014, 10(5):899-912 PMC 4223127. 
[7] Conte G, Sieira J, Sarkozy A, et al. Life-threatening ventricular arrhythmias during ajmaline challenge in patients with Brugada syndrome: incidencs, clinical feature and prognosis $[\mathrm{J}]$.Heart Rhythm, 2013,10(12):1869-1874 PMID 24055942.

[8] Dubner S, Azocar D, Gallino S, et al. Single oral flecainide does to unmask type 1 Brugada syndrome electrocardiographic pattern [J]. Ann Noninvasive Electrocardiol, 2013, 18(3):256-261 PMID 23714084.

[9] Calvo D, Rubin JM, Perez D, et al. Time-dependent response to provocative testing with flecainide in the diagnosis of Brugada syndrome [J]. Herat Rhythm, 2015, 12(2):350-357 PMID 25460174

[10] Anselm DD, Evans JM, Baranchuk A, et al. Brugada phenocopy: A new electrocardiogram phenomenon [J]. World J Cardiol, 2014, 6(3):81-86 PMID 24669289.

[11] Oreto G, Corrado D, Delise P, et al. Doulbts of the cardiologist regarding an electrocardiogram presenting QRS $\mathrm{V}_{1}-\mathrm{V}_{2}$ complexes with positive terminal wave and ST segment elevation. Conensus conference promote by the Italian Cardiology Society [J].G Ital Cardiol (Rome), 2010, 11(Suppl 2):3S-22S PMID 21361048.

[12] Antzelevitch C. J wave syndrome: Molecular and cellular mechanismus [J].J Electrocardiol, 2013, 46(6): 510-518 PMID 24011992.

[13] Razus C, Flona. M, Moga VD, et al.Early repolarization syndrome: Electroar diographic sign and clinical implications [J].Ann Noninvasive Electrocardiol, 2014, 14(1):15-22 PMID 24118137.

[14] Antzelevitch C, Yan GX. J wave syndrome [J].Heart Rhythm, 2010, 7(4): 549-558 PMID 20153265.

[15] Levy S, Sbraqia P. ECG repolarization syndrome abnormalitics (J wave syndrome) and idiopthis ventricular fibrillation [J].J Interv Card Electrophysiol, 2011, 32(3): 181-186 PMID 21833514.

[16] Mizusawa Y, Bezzina CR. Early repolarization pattern: Its ECG characteristic, arrhythmogeneity and heritability [J].J Interv Card Electrophysiol, 2014, 39(3): 185-192 PMID 24532112 .
[17] Kawata H, Morita H, Yamada Y, et al. Prognostic significance of early repolarization in inferiolateral leads in Brugada patients with documented ventricular fibrillation: A novel risk factor for Brugada syndrome with ventricular fibrillation [J]. Heart Rhythm, 2013, 10(8):1161-1168 PMID 23587501.

[18] Takaqi M, Aonumak K, Sakiquchi Y, et al. The prognostic value of early repolarization ( $\mathrm{J}$ wave) and ST-segment morphology after $\mathrm{J}$ wave in Brugada syndrome multicenter study in Japan [J].Heart Rhythm, 2013, 10(4): 533-539 PMID 23274366.

[19] Szeplaki G, Ozcan EE, Qsztheimer I, et al. Ablation of the epicardial substrate in the right ventricular outflow tract in a patient with Brugada syndrome refusing implantable cardioverter defibrillator therapy[J].Can J Cardiol,2014,30(10):1249 eg-1249 en PMID 25262866.

[20] Conte G, Sieira J, Ciconte G, et al. Implantable cardioverter-defibrillator therapy in Brugada syndrome: A 20 year single-center experience [J].J Am Coll Cardiol, 2015, 65(9): 879-888 PMID 25744001.

[21] Hsiao PY, Tien HC, Lo CP, et al. Gene mutations in cardiac arrhythmias: A review of recent evidence in ion channelopathies [J]. Appl Clin Genet, 2013, 6:1-13 PMID 23837003.

[22] Nademanee K, Veerakul G, Chandanamattha $P$, et al. Prevention of ventricular fibrillation episodes in Brugada syndrome by Catheter ablation over the anterior right ventricular outflow tract epicardium [J].Circulation, 2011, 123(12): 1270-1279 PMID 21463098.

[23] Sunsaneewitayakul B, Yao Y, Thamaree S, et al. Endocardial mapping and catheter ablation for ventricular fibrillation in Brugada syndrome [J]. J Cardiovasc Electrophysiol, 2012, 23(Suppl 1):S10-S16 PMID 22988965.

[24] Fish JM, Welchons DR, Kim Y, et al. Dimethyl lithospermate B An extract of danshen surpresses arrhythmgenesis associated with the Brugada syndrome [J]. Circulation, 2006, 113:1393-1400 PMID 16534004. 